\title{
Falla multiorgánica asociada a hipertermia en una lactante con síndrome de Prader-Willi. Caso clínico
}

\author{
Multiorgan failure associated with hyperthermia in an infant with \\ Prader-Willi syndrome. Case report
}

Dr. Alejandro Donoso F. ${ }^{a}$, Dra. Daniela Arriagada S. ${ }^{a}$, Dra. Stephanie Campbell W..$^{b}$ y Dr. Pablo Cruces R. ${ }^{a}$

\section{RESUMEN}

El golpe de calor es una emergencia médica y se debe entender como una forma de hipertermia asociada a una respuesta inflamatoria sistémica, que ocasiona falla multiorgánica y en la cual la disfunción del sistema nervioso central es predominante. Si no es tratada precozmente, puede ocasionar una alta mortalidad. El síndrome dePrader-Willi es un trastorno genético multisistémico secundario a una anormalidad en el brazo largo del cromosoma 15 (15q11-q13), caracterizado por hipotonía central neonatal, retraso del desarrollo psicomotor, hipogonadismo, hiperfagia y obesidad. Estos pacientes son proclives a presentar problemas de termorregulación. Se comunica el caso de una lactante de 5 meses en quien seestableció el diagnóstico de síndrome de Prader-Willi en el curso de un episodio febril sin foco conocido, que evolucionó con falla multiorgánica y rabdomiólisis secundaria a la hipertermia. Palabras clave: sindrome de Prader-Willi, hipertermia, golpe de calor, falla multiorgánica.

\section{ABSTRACT \\ Heat stroke is a medical emergency characterized primarily by an elevated core temperature associated with a systemic inflammatory response, which causes multiple organ dysfunction in which encephalopathy predominates. If it is not early treated has high mortality. The Prader-Willi syndrome is a multisystem genetic disorder secondary to an abnormality in long arm chromosome 15 (15q11-q13), characterized by neonatal central hypotonia, developmental delay, hypogonadism, hyperphagia and obesity. These patients are susceptible to developing thermoregulatory problems. We report the case of a 5-month-old infant, in whom a diagnosis of Prader-Willi syndrome was established in the course of a febrile episode without known focus, who developed multiorganic failure and rhabdomyolysis secondary to hyperthermia. \\ Key words: Prader-Willi syndrome, hyperthermia, heat stroke, multiorganic failure.}

http:/ /dx.doi.org/10.5546/aap.2013.e117

a. Pediatra Intensivista, Área de Cuidados Críticos, Hospital Padre Hurtado, Programa de Medicina Intensiva Infantil.

b. Facultad de Medicina Clínica Alemana.

Universidad del Desarrollo, Chile.

Correspondencia:

Dr. Alejandro Donoso F.: adonoso@hurtadohosp.cl

Conflicto de intereses: Ninguno que declarar.

Recibido:8-5-2013

Aceptado: 17-7-2013

\section{INTRODUCCIÓN}

Escasas publicaciones mencionan, entre las manifestaciones clínicas de los pacientes con síndrome de Prader-Willi, los problemas de termorregulación..$^{1-3}$ En estos pacientes, particularmente en los menores de 2 años, las enfermedades habituales de la niñez se pueden asociar con fiebre elevada y, en ocasiones, con muerte repentina. Es probable que la disfunción hipotalámica cumpla algún papel en la respuesta térmica excesiva. ${ }^{3}$

Desde el punto de vista fisiopatológico, el concepto de golpe de calor debe entenderse como una forma de hipertermia asociada a una respuesta inflamatoria sistémica que, a su vez, ocasiona falla multiorgánica y en la cual la disfunción del sistema nervioso central es predominante. ${ }^{4}$

Se comunica el caso de una lactante que presentó falla multiorgánica asociada a hipertermia, como una manifestación clínica infrecuente del síndrome de Prader-Willi.

\section{CASO CLÍNICO}

Corresponde a una niña de 5 meses, nacida a las 38 semanas de edad gestacional. El parto se realizó por cesárea debido a la posición transversa. Durante el período neonatal, estuvo hospitalizada por síndrome hipotónico, sin haberse llegado a un diagnóstico en el momento del ingreso en nuestro hospital. Consultó por historia de un día de deposiciones líquidas, sensación febril y deterioro brusco del estado general. Luego de la reanimación inicial en el servicio de urgencia regional, donde se objetivó un alza térmica de hasta $40,2^{\circ} \mathrm{C}$, la paciente fue referida a nuestro centro con el diagnóstico de shock séptico de foco abdominal. Al ingresar estaba pálida, deshidratada, mal perfundida, taquicárdica (210 lat/min), hipotensa (76/46 $\mathrm{mm} \mathrm{Hg})$ y con temperatura rectal de $39,4^{\circ} \mathrm{C}$. Se inició la reanimación con líquidos, antibioticoterapia, fármacos vasoactivos y ventilación mecánica. En los exámenes de laboratorio se destacó la presencia de acidosis 
metabólica grave ( $\mathrm{pH} 7$, bicarbonato $8 \mathrm{mmol} / \mathrm{L}$ ). Evolucionó con requerimientos de líquidos hasta $150 \mathrm{~mL} / \mathrm{kg} / 24$ horas, hemoderivados, apoyo con fármacos vasoactivos e inotrópicos, y suplementación esteroidea. Se realizó una ecografía cerebral que fue normal. Se inició ventilación de alta frecuencia oscilatoria a las 24 horas del ingreso en la Unidad de Cuidados Intensivos. La paciente presentó síndrome compartimental abdominal, falla multiorgánica y rabdomiólisis. Evolucionó con graves alteraciones en los exámenes de laboratorio y requirió peritoneodiálisis, la cual se inició el segundo día de ingresada, por la aparición de insuficiencia renal aguda con oliguria y elevación de los valores de creatinina sérica por sobre el doble de lo normal (Tabla 1). Ante la aparición de actividad convulsiva, se efectuó un electroencefalograma, que fue normal, y una tomografía computarizada cerebral, que mostró la presencia de trombosis de venas profundas y corticales con colecciones hemáticas yuxtadurales (Figura 1).
FiguRA 1. Tomografía computarizada de cerebro

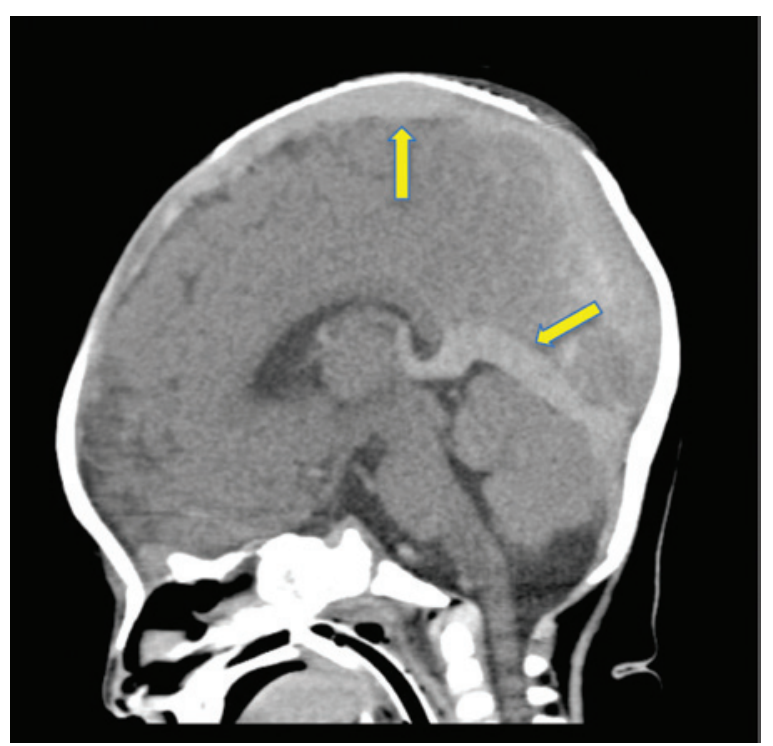

Trombosis de venas profundas y corticales con colecciones hemáticas yuxtadurales.

TABLA 1. Alteraciones de laboratorio durante la evolución de una paciente con síndrome de Prader-Willi con falla multiorgánica asociada a hipertermia

\begin{tabular}{|c|c|c|c|c|c|}
\hline & Día 1 & Día 2 & Día 3 & Día 8 & Día 15 \\
\hline Hematocrito (\%) & 23 & 30,3 & 38 & 36 & 29 \\
\hline Plaquetas $(\mu \mathrm{l})$ & 130000 & 19000 & 54000 & 116000 & 372000 \\
\hline Leucocitos $(\mu \mathrm{l})$ & 12100 & 15500 & 14100 & 14200 & 15300 \\
\hline CPK (UI/L) & 275 & 3319 & 7993 & 5150 & 968 \\
\hline $\mathrm{LDH}(\mathrm{mg} / \mathrm{dl})$ & 425 & 2336 & 1006 & 1205 & 749 \\
\hline TGOS (UI/L) & 97 & 4633 & 1651 & 223 & 38 \\
\hline TGPS (UI/L) & 36 & 1627 & 918 & 469 & 41 \\
\hline Albúmina (g/dl) & 3,4 & 2,2 & 3,4 & 4 & 3,9 \\
\hline TP (\%) & 98 & 13,4 & 13,7 & 77,1 & 95 \\
\hline TTPa (seg) & 65,5 & $>180$ & 54,2 & 33,2 & 25 \\
\hline Glucemia (mg/dl) & 65 & 276 & 192 & 94 & 131 \\
\hline Creatininemia (mg/dl) & 0,9 & 1,1 & 0,89 & 0,64 & 0,39 \\
\hline Uricemia (mg/dl) & 11,3 & 11,4 & 8,4 & 5,4 & NR \\
\hline Calcio/fósforo (mg/dl) & $7,1 / 6,6$ & $7,5 / 9,9$ & $10,6 / 3$ & $8,4 / 4,1$ & $8,6 / 3,5$ \\
\hline Sodio/potasio (meq/L) & $159 / 3,1$ & $161 / 2,7$ & $148 / 2,6$ & $149 / 3,8$ & $139 / 3,5$ \\
\hline Proteína C reactiva $(\mathrm{mg} / \mathrm{L})$ & 0,3 & 4 & 4 & 0 & 2 \\
\hline Ácido láctico (mg/dl) & 1 & 5,5 & 3,3 & 2,5 & NR \\
\hline
\end{tabular}

NR: No registrado. 
Tanto los cultivos bacterianos (hemocultivos, urocultivo y cultivos de secreción bronquial), como el estudio virológico (rotavirus, adenovirus, enterovirus y citomegalovirus) resultaron negativos. La espectrometría de masa en tándem fue normal. Por el antecedente en esta lactante de hipotonía central sin confirmación etiológica, presencia en el examen físico de dolicocefalia y dismorfia facial característica consistente en ojos almendrados, y episodio agudo de hipertermia, sin foco infeccioso demostrado, se sospechó el diagnóstico de síndrome de Prader-Willi. Este se confirmó mediante la prueba de metilación, en la que se observó la presencia de un único fragmento de $313 \mathrm{pb}$ correspondiente al alelo materno, hallazgo compatible con ese diagnóstico.

Evolucionó de manera favorable, con una mejoría progresiva después de 6 días de peritoneodiálisis. En la evaluación neurológica, una vez superado el episodio agudo, se destacó hipotonía central con motilidad y trofismo disminuidos, y reflejos osteotendinosos normales.

La paciente fue dada de alta a su domicilio después de 24 días de hospitalización, en buenas condiciones generales y con suplementación esteroidea. En los controles ambulatorios, durante los primeros 6 meses, no presentó secuelas neurológicas. Desde el punto de vista endocrinológico, se realizó la determinación de cortisol matinal, cuyo resultado fue compatible con insuficiencia suprarrenal. Quedó en control en la unidad de genética y endocrinología en su centro de referencia.

\section{DISCUSIÓN}

El síndrome de Prader-Willi tiene una incidencia estimada de $1 / 10000$ a 1/30 000 nacidos vivos. ${ }^{5}$ Las manifestaciones clínicas en el período neonatal y de lactante se caracterizan por hipotonía central grave, dificultad para alimentarse, retardo en el desarrollo e hipoplasia genital. En la adolescencia y la adultez, estos pacientes presentan problemas conductuales, obesidad por hiperfagia y retraso cognitivo. ${ }^{1}$

La base genética del síndrome de PraderWilli se encuentra en una región de 5-6 Mb en la porción proximal del brazo largo del cromosoma 15, específicamente en la región 15 q11.2-q13. ${ }^{6}$ Esta región está sometida a un sistema de control de expresión genética denominado impronta genómica. ${ }^{7}$

En el síndrome de Prader-Willi hay una pérdida de la expresión de varios genes paternos, lo cual determina su complejo fenotipo. Esta falta de expresión obedece principalmente a tres mecanismos:

1) deleción paterna del cromosoma 15 (15q11-q13), que corresponde al 65\% al 75\% de los casos,

2) disomía uniparental materna, que se observa en un $20 \%$ a $25 \%$ de los casos y que implica la herencia de dos cromosomas 15 de la madre y ninguno del padre, $y$

3) defecto en la impronta, responsable del 1\% al 3\% de los casos. Aquí los genes de origen paterno están presentes, pero inactivados. ${ }^{8}$

Más del 99\% de los casos pueden ser diagnosticados usando la prueba de metilación de DNA, ${ }^{9}$ la única técnica que permite diagnosticar el síndrome, con independencia de la alteración molecular.

Las causas de muerte en los pacientes adultos con síndrome de Prader-Willi son el cor pulmonale, relacionado con la obesidad, y la falla respiratoria hipercápnica. A su vez, en un reciente análisis de las causas de mortalidad en los niños, Tauber y cols. señalaron que las enfermedades respiratorias fueron las más frecuentes $(61 \%){ }^{10}$

Existen escasas comunicaciones de enfermedades casi letales en los niños con el síndrome de Prader-Willi, debido quizá a un diagnóstico erróneo o retardado, como lo aquí comunicado. ${ }^{1}$ La mortalidad anual de los pacientes afectados es elevada y alcanza un $3 \%$; muchas de las muertes son repentinas e inexplicadas. ${ }^{5}$

El desarrollo de episodios febriles por enfermedades intercurrentes (gastrointestinales o respiratorias), durante los primeros meses de vida, puede ocasionar alzas térmicas extraordinariamente elevadas, sin que pueda determinarse, en muchas ocasiones, su origen;, ${ }^{2,11}$ algunos de ellos conllevan un riesgo vital. ${ }^{12}$

En estos pacientes se han documentado alteraciones en el eje hipotálamo-hipófisis, ${ }^{3}$ que pueden ser la causa de los diversos mecanismos implicados en la génesis de la hipertermia, como alteración de la termorregulación, inestabilidad térmica y una sensibilidad alterada al calor durante la infancia. ${ }^{13}$ La alteración en el control de la temperatura puede ser un factor contribuyente a la gravedad y letalidad en los lactantes con síndrome de Prader-Willi. En la serie clínica de Stevenson y cols., ${ }^{1}$ todos los menores de 2 años, incluidos los fallecidos, presentaron fiebre, no así los niños mayores.

Se describió, en una casuística de pacientes 
fallecidos, ${ }^{1}$ la posibilidad de insuficiencia suprarrenal no reconocida. Asimismo, se demostró en estos pacientes, en condiciones de estrés, una incidencia del $60 \%$ de insuficiencia suprarrenal, lo que podría relacionarse con la mortalidad inesperada ante una sepsis de cualquier tipo. Estos datos apoyan el uso de hidrocortisona en las infecciones agudas. ${ }^{14}$

La paciente del caso analizado presentó un cuadro clínico de falla multiorgánica caracterizado por hipertermia (temperatura central $>40^{\circ} \mathrm{C}$ ) con una grave disfunción del sistema nervioso central, ambas características esenciales para el diagnóstico de golpe de calor, además de shock con daño hepatocelular, acidosis metabólica, rabdomiólisis, coagulación intravascular diseminada con complicaciones hemorrágicas, y alteraciones en el control glucémico. ${ }^{15}$

Aunque no se pudo demostrar una causa infecciosa, es altamente probable que las alteraciones clínicas de la paciente fueran consecuencia de la hipertermia, ya que no se observaron signos indirectos de inflamación, como ascenso de los niveles de proteína $C$ reactiva. Además, la magnitud de la elevación de las enzimas musculares y hepáticas no es frecuente de observar en los episodios de shock séptico.

El retardo en el diagnóstico puso en riesgo inminente la vida de esta paciente, pues la hipotonía y las características dismórficas presentadas desde la etapa neonatal deberían haber hecho pensar en este síndrome y así extremar las medidas destinadas a establecer un diagnóstico oportuno por parte del equipo multidisciplinario a cargo.

En conclusión, la hipertermia debe considerarse una de las manifestaciones clínicas del paciente con síndrome de Prader-Willi. Cuando aparece, se debe tener una conducta proactiva en relación con el control, la terapia y la búsqueda de complicaciones potencialmente mortales. Asimismo, se debe enfatizar la realización de una educación preventiva por parte del equipo de salud responsable del cuidado de estos pacientes hacia los padres, con respecto al alertarlos sobre el mayor riesgo de complicaciones graves ante intercurrencias banales.

\section{BIBLIOGRAFÍA}

1. Stevenson DA, Anaya TM, Clayton-Smith J, Hall BD, et al. Unexpected death and critical illness in Prader-Willi syndrome: report of ten individuals. Am J Med Genet A 2004;124A(2):158-64.

2. Ince E, Ciftçi E, Tekin M, Kendirli T, et al. Characteristics of hyperthermia and its complications in patients with Prader-Willi syndrome. Pediatr Int 2005; 47(5):550-3.

3. Swaab DF. Prader-Willi syndrome and the hypothalamus. Acta Paediatr Suppl 1997; 423:50-4.

4. Bouchama A, Knochel JP. Heat stroke. $N$ Engl J Med 2002;346(25):1978-88.

5. Whittington JE, Holland AJ, Webb T, Butler J, et al. Population prevalence and estimated birth incidence and mortality rate for people with Prader-Willi syndrome in one UK Health Region. J Med Genet 2001; 38(11):792-8.

6. Bittel DC, Butler MG. Prader-Willi syndrome: clinical, genetics, cytogenetics and molecular biology. Expert Rev Mol Med 2005;7(14):1-20.

7. Cassidy SB, Dykens E, Williams C. Prader-Willi and Angelman syndromes: sister imprinted disorders. Am J Med Genet 2000;97(2):136-46.

8. Cassidy SB, Schwartz S, Miller JL, Driscoll DJ. Prader-Willi syndrome. Genet Med 2012;14(1):10-26.

9. Zeschnigk M, Lich C, Buiting K, Doerfler W, et al. A singletube PCR test for the diagnosis of Angelman and PraderWilli syndrome based on allelic methylation differences at the SNRPN locus. Eur J Hum Genet 1997;5(2):94-8.

10. Tauber M, Diene G, Molinas C, Hébert M. Review of 64 cases of death in children with Prader-Willi syndrome (PWS). Am J Med Genet A 2008;146(7):881-7.

11. Yalçin SS, Kale E, Topalo lu H, Tunçbilek E. A hypotonic infant with tachycardia and fever of unknown origin. J Pediatr Health Care 2007;21(2):115-6,136-8.

12. Schrander-Stumpel CT, Curfs LM, Sastrowijoto P, Cassidy SB, et al. Prader-Willi syndrome: causes of death in an international series of 27 cases. Am J Med Genet A 2004;124A(4):333-8.

13. DiMario FJ Jr, Burleson JA. Cutaneous blood flow and thermoregulation in Prader-Willi syndrome patients. Pediatr Neurol 2002; 26(2):130-3.

14. de Lind van Wijngaarden RF, Otten BJ, Festen DA, Joosten $\mathrm{KF}$, et al. High prevalence of central adrenal insufficiency in patients with Prader-Willi syndrome. J Clin Endocrinol Metab 2008;93(5):1649-54.

15. Horseman MA, Rather-Conally J, Saavedra C, Surani S. A case of severe heatstroke and review of pathophysiology, clinical presentation, and treatment. J Intensive Care Med 2012 Jan 17. [Epub ahead of print]. 